\title{
A case of metastatic haemangiopericytoma to the thyroid gland: Case report and literature review
}

\author{
AGNESE PROIETTI $^{1}$, CHIARA SARTORI $^{1}$, LIBORIO TORREGROSSA ${ }^{1}$, PAOLO VITTI $^{2}$, \\ ALEKSANDR AGHABABYAN ${ }^{3}$, LORENZO FREGOLI $^{3}$, PAOLO MICCOLI ${ }^{3}$ and FULVIO BASOLO ${ }^{1}$ \\ ${ }^{1}$ Division of Pathology, Department of Surgery, University of Pisa, I-56126 Pisa; ${ }^{2}$ Department of Endocrinology; \\ ${ }^{3}$ Division of Surgery, Department of Surgery, University of Pisa, I-56124 Pisa, Italy
}

Received December 21, 2011; Accepted February 22, 2012

DOI: $10.3892 / \mathrm{ol} .2012 .661$

\begin{abstract}
Haemangiopericytoma (HPC) is a mesenchymal neoplasm accounting for a minority of all vascular tumours. HPC mostly arises in the lower extremities and the retroperitoneum, while the head and neck area is the third most common site. The majority of HPCs are histologically benign. However, a small percentage possess atypical features, such as a high mitotic rate, high cellularity and foci of necrosis. We report a case of classical abdominal HPC that presented 7 years after the first surgical resection with thyroid metastases of malignant HPC. Microscopic examination revealed multiple hypercellular nodules with an infiltrative growth pattern. These nodules consisted of tightly packed fusiform or spindle-shaped cells with nuclear polymorphism and an increased mitotic rate. The tumour cells exhibited a marked expression of CD34. Cells were arranged around a prominent vascular network, occasionally with a 'staghorn' configuration. The results of this study support and confirm the theory that HPC is a rare neoplasm with unpredictable behaviour, as largely debated in the international literature. Therefore, this study emphasized the importance of applying strict diagnostic criteria in making the most appropriate diagnosis.
\end{abstract}

\section{Introduction}

Haemangiopericytoma (HPC) is a mesenchymal neoplasm accounting for a minority of all vascular tumours. The term was first coined in 1942 by Stout and Murray for tumours thought to originate from pericytes, which are modified dendritic-like smooth muscle cell encircling blood vessels (1). According to the World Health Organisation (WHO) classification of tumours of soft tissues and bone, the term 'haemangiopericytoma' is used to refer to a variety of tumours, which have in common the presence of a thin-walled branching 'staghorn'

Correspondence to: Dr Fulvio Basolo, Division of Pathology, Department of Surgery, University of Pisa, Via Roma 57, I-56126 Pisa, Italy

E-mail: fulvio.basolo@med.unipi.it

Key words: haemangiopericytoma, thyroid, metastasis, review vascular pattern and resemble cellular areas of solitary fibrous tumours (SFTs) (2). As a result, there are difficulties in predicting clinical behaviour for a given neoplasm, and thus in establishing specific treatment modalities, since this tumour category remains to be clarified. Numerous entities have progressively escaped from the ill-defined haemangiopericytoma tumour category over the past 10 years, and those which remain tend to now be recognized as cell or malignant forms of SFT (3). Up to 15\% of soft tissue neoplasms show HPC-like features, at least focally. HPC is currently no longer considered a specific entity, but rather as a growth pattern, common to a number of often unrelated neoplasms.

HPC mostly arises in the lower extremities and the retroperitoneum, while the head and neck area is the third most common site (2). The majority of HPCs are histologically benign. However, a small percentage of HPCs possess atypical features, such as a high mitotic rate, high cellularity and foci of necrosis. Following curative surgical treatment, recurrent or metastatic tumours, or both, develop in certain patients with HPC (2). The lungs are the most common metastatic site, followed by the bones and liver. However, predicting the clinical behaviour of this tumour following the initial resection and determining the appropriate treatment for recurrent or metastatic tumours can be difficult.

We report a case of metastatic HPC in the thyroid gland and discuss the histological and immunohistochemical features and the clinical presentation.

\section{Patients and methods}

Patient. A 67-year-old male was referred to the Department of Surgery for intrathoracic multinodular toxic goiter. Seven years earlier the patient had undergone surgical resection at another hospital for an abdominal wall tumour that had been diagnosed as a classical HPC. One year prior to the referral, the patient developed lung metastases that were treated with sunitinib and radiotherapy. Preoperative CT scanning showed nodular areas in the right basal lung; the nodular areas ranged in size between 13 and $20 \mathrm{~mm}$.

At the time of thyroid surgery, the patient had hyperthyroidism (laboratory findings: TSH 3.72; fT3 1.62; fT4 0.84) and the thyroid volume was $150 \mathrm{ml}$. Radiological evaluation revealed conspicuous compression of the trachea. The thyroid 


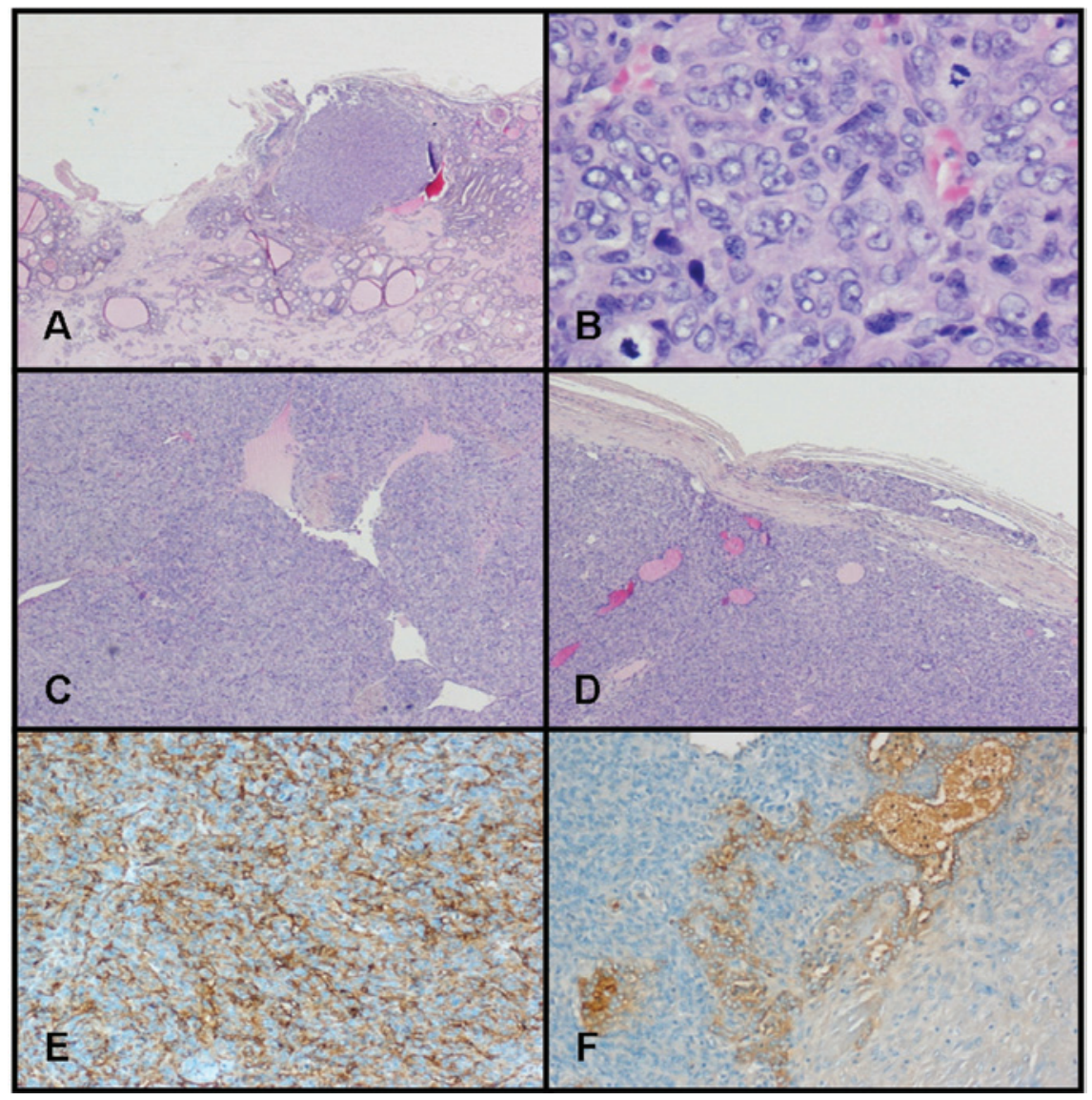

Figure 1. Present case of a 67-year-old male with HPC is shown. Haematoxylin-eosin staining showing (A) a solid nodule of spindle cells intermingled with macrofollicular thyroid parenchyma (magnification, x4); (B) the high mitotic rate (magnification, $x 40$ ); (C) tightly packed fusiform cells arranged around vascular structures with 'staghorn' configuration (magnification, $\mathrm{x} 40$ ); and (D) an embolous in the thyroid capsule (magnification, $\mathrm{x} 4$ ). Immunostain showing (E) a marked expression of CD34 (magnification, x20) and (F) thyroglobulin (magnification, $\mathrm{x} 20$ ).

gland had a prevalent left intrathoracic growth, which caused right tracheal deviation, and a total thyroidectomy was performed.

The patient is currently receiving chemotherapeutic treatment with sunitinib.

Institutional approval from the ethics committee was obtained for the study. Informed consent was obtained from the patient with regard to the use of the tumor samples.

Specimens. Specimens were surgically obtained and fixed in $10 \%$ neutral-buffered formaldehyde and embedded in paraffin. Routine haematoxylin and eosin staining was performed on the microtomic section for histopathological examination.

Immunohistochemistry. A paraffin block for immunohistochemical study was selected. Immunohistochemistry was carried out using the avidin-biotin-peroxidase complex method. Antibodies were purchased from Ventana Medical Systems (Tucson, AZ, USA). The antibodies employed included CD34, CD99, bcl-2, vimentin, cytokeratin, actin, desmin, thyroglobulin and TTF-1. All the antibodies were pre-diluted.

\section{Results}

Specimens were obtained from a 67 -year-old male who underwent thyroidectomy following examination and radical evaluation. The gross appearance of the pathological specimen was a multinodular goiter with right and left solid nodules, measuring a maximum of $5.3 \mathrm{~cm}$. Furthermore, the patient had a solid nodule in the right thyroid lodge measuring $1.1 \mathrm{~cm}$. On the cut surface, the nodules appeared as greyish masses with haemorrhagic changes. Microscopic examination revealed multiple hypercellular nodules with an infiltrative growth pattern (Fig. 1A). These nodules consisted of tightly packed fusiform or spindle-shaped cells with nuclear polymorphism. An increased mitotic rate was observed ( $>4 \mathrm{MF} / 10 \mathrm{HPF}$ ) (Fig. 1B). The cells were arranged around a prominent vasculature, which formed a ramifying network, occasionally with a 'staghorn' configuration (Fig. 1C). The vessels were lined with flat endothelial cells. Small foci of necrosis and emboli were present (Fig. 1D).

The tumour cells exhibited a marked expression of CD34 (Fig. 1E), CD99, Bcl-2, vimentin and Factor VIII, and a negative expression of thyroglobulin (Fig. 1F). Cells were negative for cytokeratins, thyroglobulin, TTF-1, actin and desmin. The proliferation marker $\mathrm{Ki}-67$ was positive in up to $60 \%$ of the tumour cells. The collateral thyroid parenchyma was multinodular.

\section{Discussion}

HPC is a rare mesenchymal tumour. According to cumulative data, it is mainly a tumour of adult patients (4), and according 
Table I. Summary of the cases with thyroidal haemangiopericytoma.

\begin{tabular}{|c|c|c|c|c|c|c|c|c|}
\hline Author & Year & Age & Gender & Side & Size $(\mathrm{cm})$ & Pleomorphism & Mitosis (MF/10 HPF) & Necrosis \\
\hline Prokš (11) & 1961 & 37 & $\mathrm{~F}$ & $\mathrm{R}$ & 6 & + & Great number & + \\
\hline Tano Assini et al (12) & 1968 & 31 & $\mathrm{~F}$ & $\mathrm{~L}$ & 4 & NR & NR & NR \\
\hline Kallenberg and Anagnostaki (13) & 1979 & 59 & M & $\mathrm{L}$ & NA & NA & NA & NA \\
\hline Tytor and Olofsson (14) & 1986 & 79 & $\mathrm{~F}$ & $\mathrm{R}$ & NR & NR & NR & NR \\
\hline Justrabo et al (15) & 1989 & 77 & $\mathrm{~F}$ & $\mathrm{~L}$ & 11.5 & - & - & - \\
\hline Geisinger et al (16) & 1990 & 66 & $\mathrm{~F}$ & NR & 8 & NR & 6 & NR \\
\hline Dictor et al (17) & 1992 & 5 & M & $\mathrm{L}$ & 8 & + & $0-1$ & - \\
\hline Cameselle-Teijeiro et al (18) & 2003 & 36 & M & $\mathrm{L}$ & 6 & NR & - & - \\
\hline Hansen et al (19) & 2004 & 15 & $\mathrm{~F}$ & $\mathrm{~L}$ & 5 & + & $4-5$ & - \\
\hline Present case & 2011 & 67 & M & LR & 7 & - & $>4$ & + \\
\hline
\end{tabular}

F, female; M, male; R, right lobe; L, left lobe; NR, not reported; NA, not available.

to Espat et al (5), the median patient age is 45 years. In their series, tumours of the extremities, pelvis, and head and neck occurred with similar frequency. Extremity tumours were mostly in the axilla and thigh, and the head and neck group accounts for tumours of the meninges and of the cheek (5).

Pain is a late symptom, associated with an enlarging mass; however, specific symptoms are associated with the location of the tumour. Hypoglycemia has been reported in approximately $5 \%$ of HPC, most often located in the pelvis and retroperitoneum. In 1990, Benn et al showed that hypoglycemia is mediated by the production of insulin-like growth factor by the tumour (6).

HPC grows in deep soft tissues as a circumscribed brown lesion surrounded by a pseudo-capsule, or as an exophytic lesion from the serosal surfaces. HPCs are extremely variable in appearance, depending on the relative proportion of cells and fibrous stroma. Classical HPC consists of tightly packed round to fusiform cells, arranged around an elaborate vasculature. The vascular network is ramified and shows variation in calibre, with a 'staghorn' configuration. Frequently the vessels are surrounded by a thick coat of collagen, which extends into the interstitium. Enzinger and Weiss suggest that when the prevalent component of the tumours are spindle cells arranged in short, ill-defined fascicles, with a striking hyalinisation, the lesions should be recognized as classical solitary fibrous tumours (2). The criteria for malignancy proposed by Enzinger and Smith in classical HPC identify overtly malignant or high-grade lesions, but fail to address low-grade lesions (7). In their study, large-sized tumours $(>5 \mathrm{~cm})$, increased the mitotic rate ( $\geq 4 \mathrm{MF} / 10 \mathrm{HPF}$ ), high cellularity, presence of immature and pleomorphic tumour cells, and foci of haemorrhage and necrosis predicted a highly malignant course. Enzinger and Weiss employed the term 'low-malignant potential' for lesions with lower levels of mitotic activity (1-3 MF/10 HPF), particularly if they have any degree of atypia and cellularity (2). However, it should be emphasized that a number of cases exhibit high-grade features. Numerous HPCs express CD34; however, desmin and cytokeratin are usually absent (3).

The inability to render an accurate assessment of the biological potential of the tumour is a significant problem for pathologists. At present, no prognostic criteria exist for which there is universal agreement. However, the prognosis of HPC is generally favourable, although recurrent or metastatic tumours, or both, develop in some patients following curative surgical treatment (2). In 2002, Espat et al reported a 93 and $80 \%$ 2- and 5-year survival rate, respectively, for classical HPC (5). It was noted that all patients undergoing complete surgical excision were alive at 5 years after surgery. Significantly higher disease-free survival and lower local recurrence rates were associated with extremity lesions versus meningeal and retroperitoneal lesions. In 1998, Spitz et al (8)reported a local failure rate of $32 \%$ in patients treated with curative intent. However, these authors suggested that local recurrence is not an indicator of poor prognosis in these patients (8). Frequently, patients develop metastases together with the primary tumour. Classical HPC has a low diseaseassociated mortality, whereas malignant tumours have a more variable outcome. Approximately one-half of the patients were cured of their tumours following excision, whereas the remainder developed recurrence, metastases or both (2). Metastases were noted in approximately $30 \%$ of patients, with a 5-year actuarial survival of $71 \%$ (8). The most common metastatic sites were the lungs, bones and liver.

HPC is a rare tumour of the thyroid and can invade neighbouring tissues. Primary diagnosis with fine-needle aspiration biopsy (FNAB) is extremely difficult; however, it has a characteristic and easily diagnosed histopathological appearance.

In this study, we report a case of classical abdominal HPC that presented 7 years after the first surgical resection with thyroid metastases of malignant HPC. A review of the literature (Table I) regarding HPC of the thyroid gland showed that it is extremely rare (9-10), with only 9 cases being reported thus far (11-19).

According to the cumulative data, including the present case, the mean age of the patients is 47 years (range, 5-79). There is a predominant incidence in females, with a female:male ratio of $3: 2(\mathrm{~F}=6 ; \mathrm{M}=4)$. The mean size of the tumours is $7 \mathrm{~cm}$ (range, 4-11.5). Six tumours (77\%) were located in the left lobe, one case was bilateral and in one case the location of the lesion was not reported. The reported cases were primitive thyroid gland 
HPCs with at least one histological feature (pleomorphism, mitosis rate or necrosis) suggestive for potentially malignant behaviour, with the exception of the case studies by CameselleTeijeiro et al (7), which was a lipomatous HPC, and that of Justrabo et al (15), which was a benign HPC. No evidence of local recurrence or distant metastases was reported.

Our case showed an uncommon location of a metastatic classical HPC, which recurred following surgical treatment and chemo-radiotherapy. In the metastatic site, the tumour acquired the histological features of a malignant HPC. We can speculate as to how a classical HPC with clinically aggressive behaviour may be associated with malignant histological changes. Furthermore, we support the study by Espat et al, who, after observing a mortality rate of greater than $50 \%$ in patients affected by conventional HPC (5), emphasized the importance of applying strict diagnostic criteria in making the most appropriate diagnosis.

\section{Acknowledgements}

This study has been supported in parts by grants from the Ministero dell'Istruzione, dell'Università e della Ricerca, Associazione Italiana per la Ricerca sul Cancro, Istituto Toscano Tumori and Ministero della Salute.

\section{References}

1. Stout AP and Murray MR: Hemangiopericytoma: a vascular tumor featuring Zimmermann's pericytes. Ann Surg 116: 26-33, 1942.

2. Enzinger FM and Weiss SW (eds): Soft Tissue Tumors. 5th edition. Mosby, New York, NY, 2008.

3. Gengler $C$ and Guillou L: Solitary fibrous tumour and haemangiopericytoma: evolution of a concept. Histopathology 48: 63-74, 2006.

4. Enzinger FM and Weiss SW (eds): Soft Tissue Tumors. 3rd edition. Mosby, New York, NY, 1995.
5. Espat NJ, Lewis JJ, Leung D, Woodruff JM, Antonescu CR, Shia J and Brennan MF: Conventional hemangiopericytoma. Cancer 95: 1746-1751, 2002.

6. Benn JJ, Firth RG and Sönksen PH: Metabolic effect of an insulin-like factor causing hypoglycemia in a patient with haemangiopericytoma. Clin Endocrinol 32: 769-780, 1990.

7. Enzinger FM and Smith BH: Hemangiopericytoma. An analysis of 106 cases. Hum Pathol 7: 61-82, 1976.

8. Spitz FR, Bouvet M, Pisters PW, Pollock RE and Feig BW: Hemangiopericytoma: a 20-year single-institution experience. Ann Surg Oncol 5: 350-355, 1998.

9. Brandwein MS, Kapadia SB and Gnepp DR: Nonsquamous pathology of the larynx, hypopharynx, and trachea In: Diagnostic Surgical Pathology of the Head and Neck. Gnepp DR (ed). Saunders, Philadelphia, PA, pp304-305, 2001.

10. Weis SW and Goldblum JR (eds): Soft Tissue Tumors. Mosby, St. Louis, 2001.

11. Prokš C: Generalized hemangiopericytoma of the thyroid gland (report of a case). Neoplasma 8: 219-224, 1961.

12. Tano Assini MT, Oliva Otero G and Gomez SC: Visceral hemangiopericytoma. Presentation of 2 cases. Prensa Med Argent 55: 996-1000, 1968.

13. Kallenberg F and Anagnostaki L: Hemangiopericytoma of the thyroid gland. Ugeskr Laeger 141: 3530-3531, 1979.

14. Tytor M and Olofsson J: Thyroid tumors invading the larynx and trachea. J Otolaryngol 15: 74-79, 1986.

15. Justrabo E, Michiels JF, Maire J, Jacquot JP and Levillain P: Hemangiopericytoma of the thyroid gland. Ann Endocrinol (Paris) 50: 26-30, 1989.

16. Geisinger KR, Silverman JF, Cappellari JO and Dabbs DJ: Fineneedle aspiration cytology of malignant hemangiopericytomas with ultrastructural and flow cytometric analyses. Arch Pathol Lab Med 114: 705-710, 1990.

17. Dictor M, Elner A, Andersson T and Fernö M: Myofibromatosislike hemangiopericytoma metastasizing as differentiated vascular smooth-muscle and myosarcoma. Myopericytes as a subset of 'myofibroblasts'. Am J Surg Pathol 16: 1239-1247, 1992.

18. Cameselle-Teijeiro J, Manuel Lopes J, Villanueva JP, Gil-Gil P and Sobrinho-Simões M: Lipomatous haemangiopericytoma (adipocytic variant of solitary fibrous tumour) of the thyroid. Histopathology 43: 406-408, 2003.

19. Hansen T, Gaumann A, Ghalibafian M, Höferlin A, Heintz A and Kirkpatrick CJ: Haemangiopericytoma of the thyroid gland in combination with Hashimoto's disease. Virchows Arch 445: 315-319, 2004. 